\title{
Research of psychological features of the personality of students exhibiting addictive behavior
}

\author{
Natalia N. Bessonova ${ }^{1}$, Vera S. Butenko ${ }^{2}$, Natalia A. Gudz ${ }^{3}$, Anna M. Kukulyar ${ }^{4 *}$ \\ ${ }^{1}$ «Elephant»,344010 Rostov-on-Don, Russian Federation \\ ${ }^{2}$ Rostov State Medical University, 344022 Rostov-on-Don, Russian Federation \\ ${ }^{3}$ Maikop city polyclinic №2, 385000 Maykop, Russian Federation \\ ${ }^{4}$ Southern federal university, Academy of psychology and pedagogy, 344006 Rostov-on- \\ Don, Russian Federation
}

\begin{abstract}
Annotation. This article is devoted to the study of psychological characteristics of the personality of students experiencing withdrawal syndrome from amphetamine-type psychostimulants. The study was conducted on 45 young men, students of 1-3 courses of universities of Rostov-on-don, rehabilitated In the center of psychological correction "Elephant". During the study, we used the following methods: 1) the scale of "Amphetamine Cession Symptom Assessment" (ACSA), 2) the scale of Montgomery and Asberg (MADRS); 3) the scale of EuroQol; 4) the scale of self-report (VAS). The results obtained allow us to conclude that among young men with amphetamine dependence accompanied by withdrawal syndrome in the structure of which affective rather than somatovegetative manifestations predominate almost in the complete absence of algic phenomena. Affective disorders diagnosed in the hospital center of the respondents, mainly manifested in the form of depression. The maximum expression of these States, as well as uncontrolled attraction to surfactants was achieved in the interval from the third to the seventh day in withdrawal syndrome. Further the condition of rehabilitators was normalized from the first week and till the twenty-first day of stay. We noted a similar dynamics in assessing the state of their own well-being rehabilitated.
\end{abstract}

\section{Introduction}

In modern transitive society there are radical changes in the structure of society, interpersonal relationships, value-semantic structure of the person, directly affecting the Commission of risky actions by the individual. The realities of modern society and various social problems encourage the person to exercise various destructive behaviors in reaction to frustrating events, uncertainty, stressors, caregiving from a traumatic reality (T. V. White, T. G. Bohan, A. Y. Egorov, A. O. Kibito, M. M. Orlov, E. N. Skripacheva, O. N.

\footnotetext{
${ }^{*}$ Corresponding author: vetkina-anna@mail.ru
} 
Tkachev, A. V. Trusova) [1,3]. Man has become quite often to show such protective behavior in situations related to life crises, which entails the emergence of various forms of dependent behavior from completely different additive agents (alcohol, drugs, information dependence, gambling, etc.). In this article, we would like to draw attention to the growing manifestation of such forms of addictive behavior as the use of amphetamine-type psychostimulants ("salts") among young people, and students in particular.

\section{Discussion}

Specificity of action of psychostimulants of this type at the level of physiology consists in release of neurotransmitters which are capable to provide chemical transfer of a nervous impulse in CNS, on a par with it process of slowdown of their reuptake is carried out. When using psychostimulants, a person experiences General stimulation of VPF, accelerates thinking processes and cognitive activity, retreats apathy, fatigue and a feeling of drowsiness, increases efficiency, increases the level of emotional background and sociability, improves mood, also against the background of toning the entire muscular system of the body improves coordination, a strong increase in endurance and physical strength [2].

As a consequence, the use of amphetamine-type psychostimulants by young people results in a surge of vivacity and energy, a General lifting of physical strength, the removal of fatigue and a partial improvement of such intellectual abilities as memory, attention and thinking processes, as well as mood enhancement, i.e., an optimal physiological and psychological state is achieved from their point of view $[4,5]$.

To date, in the youth subculture, the use of these psychostimulants is very common. This is primarily due to the easy availability of their purchase. With the development of social networks, in particular The Telegram, there was no need for personal meetings to buy drugs. At the moment within many SOC. there are closed communities for the sale and distribution of drugs, even work related to the implementation of "bookmarks" for potential buyers. Thus, the trade in illicit drugs has long moved to a new level. Secondly, amphetamine-type psychostimulants are available from a financial point of view, in relation to other drugs. Third, these psychostimulants are "light" drugs, since they can be used sporadically: when visiting Nightclubs, parties, etc.

Due to the relevance of the topic, we examined students of 1-3 courses in the amount of 45 people. The main part of the sample consisted of young men-students who took a course of rehabilitation measures In the center of psychological correction «Elephant» (hereinafter rehabilitated) from 18 years to 21 years with a diagnosis of "syndrome of dependence on psychostimulants (amphetamine/methamphetamine)".

The main criteria for inclusion in the study were rehabilitated: 1) the fact of the use of surfactants (amphetamine / methamphetamine) for at least 10 days in the last month before admission To the center "Elephant»; 2) confirmed diagnosis of psychostimulant dependence syndrome (amphetamine/methamphetamine) according to ICD-10;3) no use of other surfactants before the start of rehabilitation activities for at least 10 days.

Just note the criteria for which rehabilitated excluded from the study: 1) the presence of a syndrome of dependence on other surfactants, except nicotine; 2) any mental illness identified in the course of the study; 3) identification of suicidal tendencies; 4) diagnosis of severe comorbidity, which requires hospitalization in the specialized Department.

\section{Research methods and techniques}


The respondents were selected on the basis of a list of specified criteria and the compliance of the rehabilitated with them. Inclusion in the study group was based on the time sequence of admission to the center of psychological correction "Elephant". It should be noted that upon arrival at the Center, each of the participants was subjected to a thorough examination (surrendered: General blood and urine tests, biochemical tests, as well as tests to determine the presence of traces of drugs in the urine). A mandatory requirement for determining rehabilitated in the Center, was the presence of the conclusion of the therapist or other specialized specialists (neurologist, surgeon, infectious diseases, etc.).

During the research we used the following methods: 1) scale "Amphetamine Cessation Symptom Assessment" (ACSA), assessing the duration and severity of withdrawal syndrome of psychostimulants; 2) the dynamics of depressive disorders was estimated by us with the help of the scale Montgomery and Asberg (MADRS); 3) assessment of quality of life was performed using the EuroQol scale; 4) evaluation of the craving for stimulants was carried out on $100 \mathrm{~mm}$ visual-analog scale self-report (VASS).

During the study, according to the scale "Amphetamine Cession Symptom Assessment" (ACSA) and the Montgomery and Asberg scale (MADRS), we conducted measurements on the first, third, seventh, tenth and twenty-first day, in order to identify the dynamics of the duration and severity of psychostimulant withdrawal syndrome and the specifics of the course of depressive disorder. The quality of life, studied on the EuroQol scale, was diagnosed once a week according to the inpatient stay at the Center, and then every 4 weeks on an outpatient basis. The degree of attraction to psychostimulants on the VASH scale was carried out every 3 days within the framework of the withdrawal syndrome, then 1 time per week in the hospital and 1 time per week with outpatient observation.

\section{Research results}

During the collection of anamnestic data, when rehabilitating in the Center within the hospital, we obtained the following results. In persons with identified amphetamine dependence, in the study of the characteristics of the course of the withdrawal syndrome, affective disorders were diagnosed. The presence of these violations was noted in all 45 boys ( $100 \%$ of the total sample). Along with this, $89 \%$ of respondents (41 boys) identified somatovegetative disorders. The data obtained are reliable at $\mathrm{p}<0.05$.

We also studied the specifics of the manifestation of affective disorders. In the course of the study, we found that the prevailing in the structure of affective disorders during withdrawal syndrome in persons with amphetamine dependence was a reduced emotional background- $62.4 \%$ of respondents. Irritability, as a manifestation of affective disorder, accounts for $28.8 \%$ (13 boys). Increased emotional background was diagnosed only in $8.8 \%$ of respondents (4 boys).

According to the data obtained on the MADRS scale, regarding the dynamics of affective disorders, it can be concluded that the average total index for the first two weeks of stay in the hospital of the Center in the entire group of respondents is 16.2 , which exceeds the permissible norm (15). The obtained indicator indicates the presence of depressive States in the study group of young men. We consider it important to note that the highest values on the MADRS scale (from 18.48 to 18.71) were obtained in the interval from the third to the seventh day after the cancellation of surfactants. After 10 days of stay in the hospital of the Center, there is a decline in the manifestation of depressive States, and upon reaching the twenty-first day, these manifestations among the group of young men are completely stopped.

When processing the average data on the MADRS scale, we obtained significant differences at $\mathrm{p}<0.05$ for the indicators of the first and third days (16.2 and 18.48), as well as between the indicators of the seventh and twenty-first days (18.71 and 14.0) at $\mathrm{p}<0.05$. 
We would like to note the fact that the analysis of somatic manifestations in withdrawal syndrome showed almost complete absence of any complaints, as only 3 young men (6.7\%) felt pronounced pain.

The presence of somatovegetative manifestations of withdrawal syndrome and affective disorders in rehabilitated have a negative impact on their assessment of the quality of life. According to the results of the study obtained on the EuroQol scale, within 1 day of stay in the hospital of the center, rehabilitees assess their condition by $67.29+-0.78$ points on a visual 100-point scale. By the end of the first week of stay in the hospital Center with the cancellation of amphetamine average score reached 53.38+-2.57. The presented dynamics of the results is reliable at $\mathrm{p}<0.05$. After passing stationary events during the 3 weeks the average assessment of quality of life in reabilitologist boys reached 80,91+-1,92. During the outpatient observation of the respondents, we noted a significant increase in the average on this scale to $92.02+-1.97$ points. These results can be regarded as an improvement in the dynamics of well-being among young men who were able to refrain from using amphetamine-type psychostimulants for a longer period.

The second block of the EuroQol scale helped us to study in detail the question of which predictors have the greatest impact on the self-esteem of the rehabilitated. Note that the mobility of the majority of subjects did not suffer in the first days of rehabilitation (the absence of such a problem was noted by $90-93 \% \%$ of persons), and within 14 days all rehabilitated mobility was within the norm.

From Fig. 1 we see that in the first day of cancellation 11\% (5 people) noted a decrease in activity and $11 \%$ (5 people) complained of a sharp decrease in activity. However, on the 7 th day, the total number undergoing rehabilitation activities and complaining of feeling made up almost half $(48 \%)$ of the subjects. By the 14 th day, the total number of people who noticed a decrease in activity was 24 people $(53 \%)$. But by the 21 st day, the number of people who noted a decrease in activity was only 4 people $(9 \%)$.

I would like to note the fact that among the entire group of young men at the stage of admission to the hospital Center and during the first day of pain in acute abstinence was expressed quite weakly only in $17.7 \%$ of respondents ( 8 boys), of which only $6.6 \%$ ( 3 boys) manifested significantly. After the first week, the pain symptom in the form of General malaise was diagnosed in only $11 \%$ (5 boys). After two weeks, only $4.4 \%$ ( 2 boys) showed some discomfort. By the end of the third week, only $1 \%$ (1 youth) experienced minor symptoms of discomfort. Assessing the self-esteem of respondents, we were diagnosed with anxiety, which were observed in 28 boys $(62 \%)$ and almost all of them (26 boys - $58 \%$ ) reached a significant degree of severity.

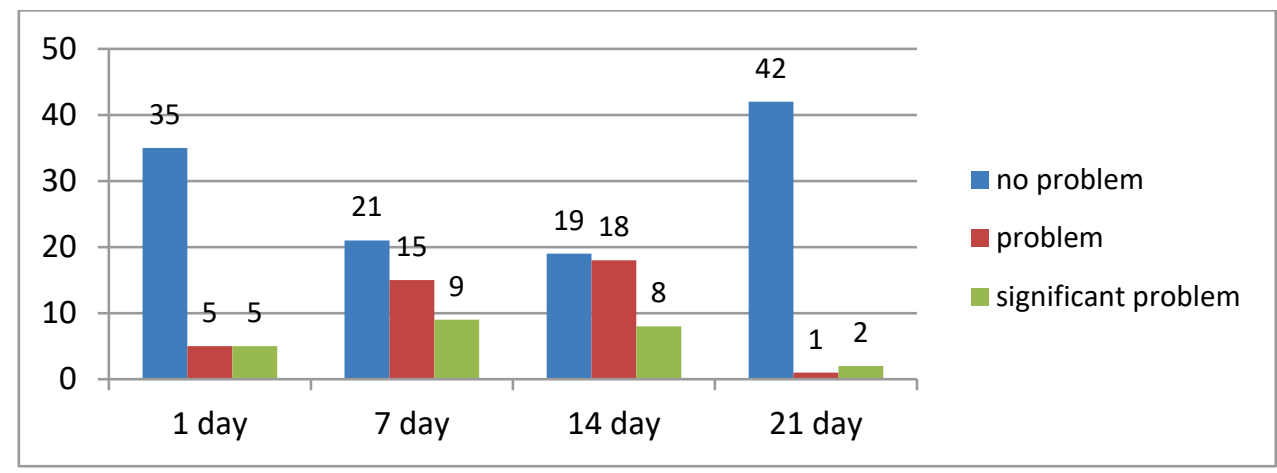

Fig. 1. Dynamics of self-evaluation activity reabilitologist within the cancellation period amphetamines (in\%) 
By the 14th day, the number of respondents experiencing anxiety decreased to 26 , and by the end of the third week, 91.1\% (41yunosh) felt the absence of any problems in this area.

Using the scale "Amphetamine Cession Symptom Assessment" (ACSA) there was an assessment of the severity and duration of withdrawal syndrome from psychostimulants. As a result of gamia, it was revealed that the most severe symptoms when canceling surfactant are observed on the third day. Significant relief of symptoms was observed only at the onset of the tenth day of stay in the hospital Center (Fig. 2).

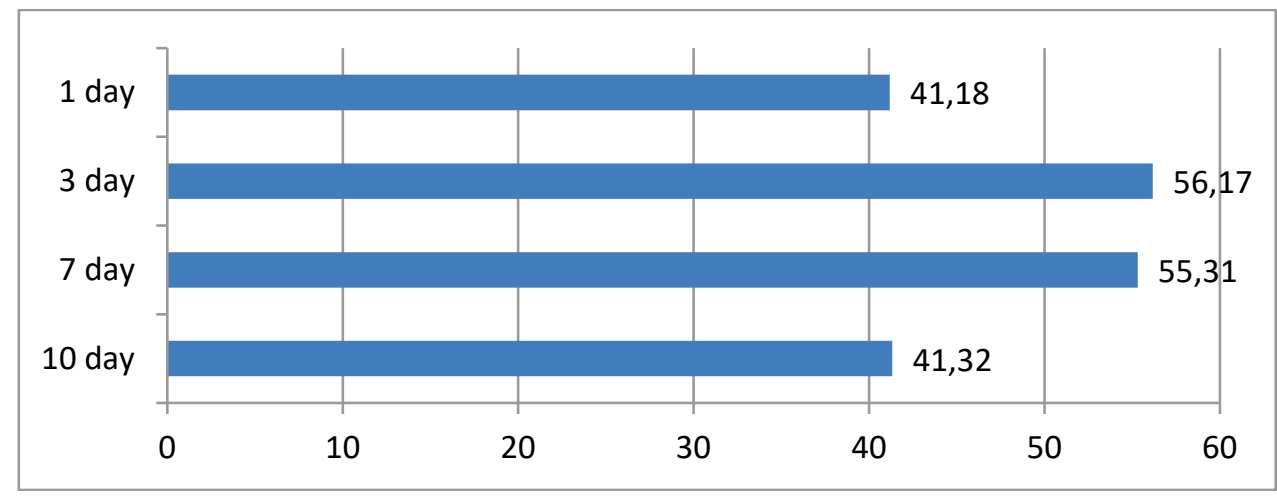

Fig. 2. Dynamics of ACSA scale indices in rehabilitated patients with amphetamine dependence

We were also interested in studying the dynamics of amphetamine cravings among the young men surveyed, which we identified using the ACSA scale. The study found that the most pronounced attraction to amphetamine-type psychostimulants was observed on the third day of hospital stay at the Center. The decrease in craving for amphetamine-type surfactants was observed only by the tenth day of being in the Center (Fig. 3). The presented results are reliable at $\mathrm{p}<0.05$.

The analysis of the results on the VASS scale gave us the opportunity to describe in more detail the specifics of the dynamics of attraction to amphetamine-type psychostimulants among young men. Note that with withdrawal syndrome, the maximum attraction to psychostimulants was recorded on the third day.

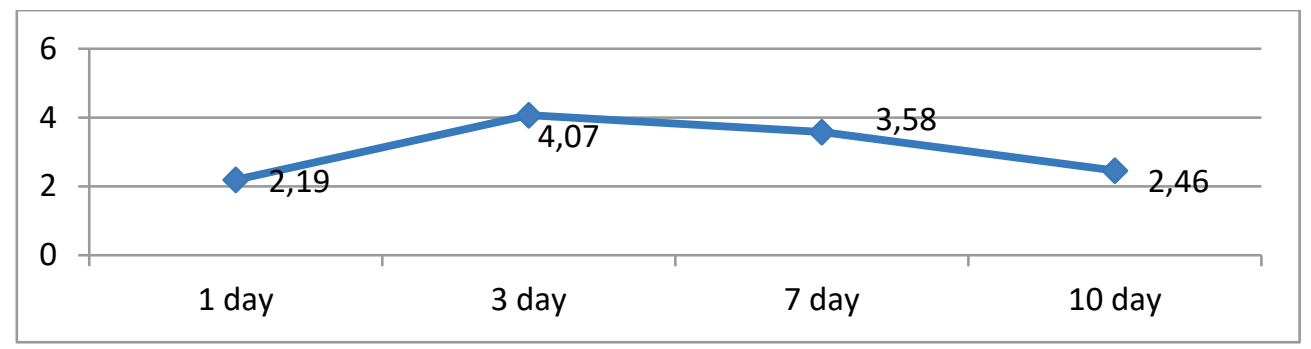

Fig. 3. Dynamics of craving for amphetamines (average score on a scale of ACSA)

At the end of the first week in the hospital, this attraction in young men began to decline. When diagnosing the dynamics of attraction to amphetamines from the tenth to the twentyfirst day of stay in the hospital of the Center, young men noted a persistent decrease in traction to a minimum (Fig. 4). 


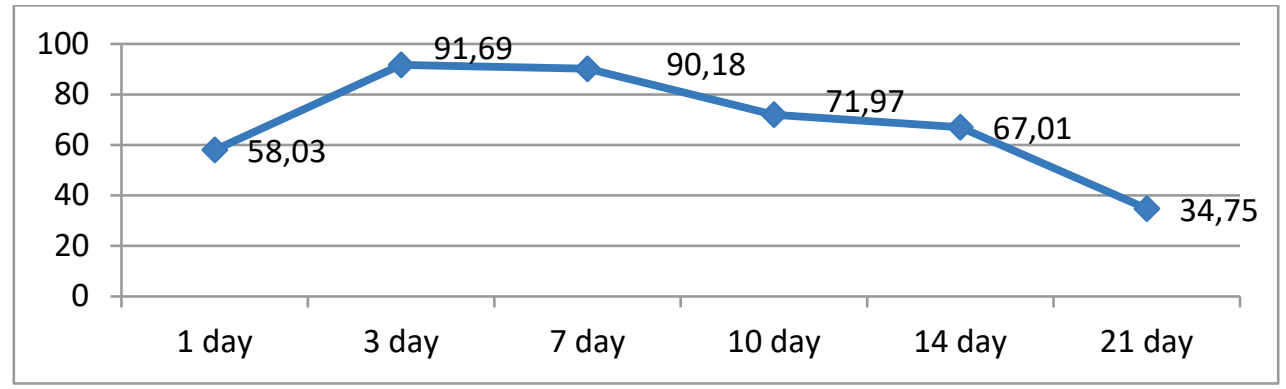

Fig. 4. The dynamics of attraction to amphetamines in the framework of the syndrome in individuals with amphetamine dependence (the average score for VASS)

\section{Conclusion}

Thus, it can be concluded that among the young men participating in the study was diagnosed amphetamine dependence accompanied by withdrawal syndrome in the structure of which affective rather than somatovegetative manifestations predominate in the almost complete absence of algic phenomena. Affective disorders diagnosed in the hospital center of the respondents, mainly manifested in the form of depression. The maximum expression of these States, as well as uncontrolled attraction to amphetamine-type psychostimulants was achieved in the interval from the third to the seventh day in withdrawal syndrome. Further after finding within a week and till the twenty-first day there was a gradual normalization of a condition of young men. We noted a similar dynamics in assessing the state of their own well-being rehabilitated. The worst was from the third to the seventh day, and then there was a significant improvement by the twenty-first day.

After passing the inpatient rehabilitation program, the subjects underwent an outpatient program, during which studies were also conducted to determine the state of health. We noted that by the 100th day of abstinence from the use of surfactants, the health of the subjects almost normalized. Significant conditions determined by dysphoria in people who refused to take surfactants are decreased activity, anxiety, depression. With all this, it should be emphasized that the subjects noted a slight degree of discomfort. Refusal to use amphetamines for a period of 3 months or more as a result of hospital stay in the center occurred in $72 \%$ of rehabilitated (in the first year of remission).

\section{References}

1. A.E. Primerova, Young scientist. 2, 1000-1003 (2016)

2. A.A. Yusifova, V.S. Knyazev, Advances in modern natural science. 6, 96-97 (2014)

3. «Amphetamine gets the job done: Using drugs to work long hours» (ScienceDaily, 2015) www.sciencedaily.com/releases/2015/02/150220083916.htm (accessed August 19, 2019)

4. W. Pedersen, S. Sandberg, H. Copes, Deviant Behavior. 36 (2), (2014)

5. V. White, T. Williams, Australian secondary school students' use of tobacco, alcohol, and over-the-counter and illicit substances in 2014 (The Cancer Council, Melbourne, 2016) 